\title{
Cooperative Adaptive Time-Varying Formation Tracking for Multi-Agent Systems with LQR Performance Index and Switching Directed Topologies
}

DOI:

10.1109/CDC.2018.8619623

\section{Document Version}

Accepted author manuscript

Link to publication record in Manchester Research Explorer

Citation for published version (APA):

Hu, J., \& Lanzon, A. (2019). Cooperative Adaptive Time-Varying Formation Tracking for Multi-Agent Systems with LQR Performance Index and Switching Directed Topologies. In Proceedings of the 57th IEEE Conference on Decision and Control, Miami, FL, USA, Dec 2018, https://doi.org/10.1109/CDC.2018.8619623

Published in:

Proceedings of the 57th IEEE Conference on Decision and Control, Miami, FL, USA, Dec 2018,

\section{Citing this paper}

Please note that where the full-text provided on Manchester Research Explorer is the Author Accepted Manuscript or Proof version this may differ from the final Published version. If citing, it is advised that you check and use the publisher's definitive version.

\section{General rights}

Copyright and moral rights for the publications made accessible in the Research Explorer are retained by the authors and/or other copyright owners and it is a condition of accessing publications that users recognise and abide by the legal requirements associated with these rights.

\section{Takedown policy}

If you believe that this document breaches copyright please refer to the University of Manchester's Takedown Procedures [http://man.ac.uk/04Y6Bo] or contact uml.scholarlycommunications@manchester.ac.uk providing relevant details, so we can investigate your claim.

\section{OPEN ACCESS}




\title{
Cooperative Adaptive Time-Varying Formation Tracking for Multi-Agent Systems with LQR Performance Index and Switching Directed Topologies
}

\author{
Junyan $\mathrm{Hu}$ and Alexander Lanzon
}

\begin{abstract}
This paper presents a novel distributed adaptive time-varying formation tracking protocol for general linear multi-agent systems. In contrast to the existing distributed methods that require global information of the interaction graph, the proposed control strategy is fully distributed such that each agent only requires its own information and the information from its neighbors through switching directed communication networks. Then, an algorithm to determine the control parameters is presented, where feasible formation condition for the followers to accomplish the desired timevarying formation tracking is provided. Furthermore, the proposed strategy is also guaranteed to achieve an optimal control performance index by using the inverse optimal approach. Simulation results are provided to verify the effectiveness of the proposed strategy.
\end{abstract}

\section{INTRODUCTION}

In recent years, distributed cooperative control of multiagent systems have received significant attention from both the practical engineering and academic communities due to their broad prospect in applications, such as unmanned aerial vehicles formation, multi-robot cooperation, distributed sensor networks, etc. The research field includes consensus control [1], rendezvous control [2], containment control [3], and formation control [4]. Formation control of multi-agent systems is hence a key active area of research, which has experienced a rapid growth in the research efforts from the international robotics community. Three main approaches of formation control, namely, leader-follower based approach, behaviour based approach, and virtual structure based approach, are unified in the framework of consensus problems as shown in [5].

Recently, with the development of algebraic graph theory, many consensus-based control methods have been applied to solve formation control problems. Motion trajectory tracing and formation control of first-order and second-order multirobot systems are presented in [6] and [7], respectively. It is worth emphasizing that the dynamics of each agent can only be described by high-order system in some practical applications. [8] discusses the formation stability problems for general high-order swarm systems, and the result is

\footnotetext{
*This work was supported by the Engineering and Physical Sciences Research Council (EPSRC) [grant number EP/R008876/1]. All research data supporting this publication are directly available within this publication.

The authors are with the Control Systems Centre, School of Electrical and Electronic Engineering, University of Manchester, Manchester, M13 9PL, UK. (e-mail: Junyan.Hu@manchester.ac.uk, Alexander.Lanzon@manchester.ac.uk)
}

extended to deal with formation tracking for multiple highorder autonomous agents by using a two-level consensus approach in [9]. Static formation experiments on quadrotor swarm systems based on consensus strategies is achieved in [10], while time-varying formation control of multi-agent systems is still a vigorously active research topic with much progress still needed.

As a matter of fact, time-varying formation will bring the derivative of the formation information to the analysis, which presents a significant challenge to control system design. Time-varying formation tracking analysis and design for second-order and high-order multi-agent systems are presented in [11] and [12], respectively. Furthermore, an application to unmanned aerial vehicles is investigated in [4]. However, in the aforementioned results, it is required to know the smallest positive eigenvalue of the Laplacian matrix of the graph topology before designing the controller, which means the control strategies are not fully distributed. In order to overcome the limitation of requiring global information of the interaction topologies, distributed adaptive control methods have been developed by researchers in recent years, which only rely on local information and information from its neighbors. The distributed adaptive control protocol proposed in [13] requires a fixed undirected communication graph, which is not robust and reliable when communication failure occurs. Due to the presence of communication channel disconnection in practical application, it is meaningful to study time-varying formation control with switching directed interaction topologies of networked systems.

Motivated by the challenges stated above, a fully distributed adaptive time-varying formation control protocol for general linear multi-agent systems in switching directed graphs is proposed. Compared with previous results on formation control, the contributions in the study can be summarized as follows:

1) A fully distributed time-varying formation tracking protocol for general multi-agent systems with switching directed communication networks is first proposed, where no global information of the communication topologies is required.

2) In our proposed design, the adaptive control scheme is also extended to achieve Linear Quadratic Regulator (LQR) performance index of multi-agent systems by solving algebraic Riccati equations, which ensures the performance of the global system. 
The paper is organized as follows. Notation and preliminaries on algebraic graph theory are presented in Section II. Distributed adaptive time-varying formation control protocol is designed in Section III. The proposed adaptive control protocol is proved to minimize certain global LQR performance index in Section IV. Simulation results are provided to verify the effectiveness of the proposed controller in Section V. Conclusions are given in Section VI.

\section{PRELIMINARIES}

\section{A. Notation and Definitions}

Let $I_{n} \in \mathbb{R}^{n \times n}$ denote the identity matrix of dimension $n$ and $\mathbf{1}_{N} \in \mathbb{R}^{N}$ be the vector with all entries equal to one. $\operatorname{diag}\left\{a_{i}\right\}$ represents a diagonal matrix with diagonal entries $a_{i}$. The Kronecker product is denoted by $\otimes$.

\section{B. Graph Theory}

Consider a weighted and directed graph $\mathcal{G}=(\mathcal{V}, \mathcal{E}, \mathcal{A})$ with a nonempty set of $N$ nodes $\mathcal{V} \in\{1,2, \ldots, N\}$, a set of edges $\mathcal{E} \subset \mathcal{V} \times \mathcal{V}$, and associated adjacency matrix $\mathcal{A}=$ $\left[a_{i j}\right] \in \mathbb{R}^{N \times N}$. An edge rooted at node $i$ and ended at node $j$ is denoted by $(i, j)$, which means information can flow from node $i$ to node $j . a_{i j}$ is the weight of edge $(j, i)$ and $a_{i j}>0$ if $(j, i) \in \mathcal{E}$. Assume that there are no repeated edges and no self loops. Node $j$ is called a neighbour of node $i$ if $(i, j) \in$ $\mathcal{E}$. Define the in-degree matrix as $D=\operatorname{diag}\left\{d_{i}\right\} \in \mathbb{R}^{N \times N}$ with $d_{i}=\sum_{j=1}^{N} a_{i j}$. The Laplacian matrix $L \in \mathbb{R}^{N \times N}$ of $\mathcal{G}$ is defined as $L=D-\mathcal{A}$. A directed graph has or contains a directed spanning tree if there exists a node, called the root, such that there exists a directed path from this node to every other nodes.

The interaction topology of the multi-agent system is assumed to be switching. Let $\left[t_{p}, t_{p+1}\right]$ be an infinite sequence of uniformly bounded non-overlapping time interval, with $t_{1}=0,0<\tau_{0} \leq t_{p+1}-t_{p} \leq \tau_{1}$. The communication topology changes at the switching sequence $t_{p}$ and remains fixed during the time interval $\tau_{0}$. Let $\eta:[0,+\infty) \rightarrow$ $\{1, \ldots, p\}$ be a switching signal whose value at time $t$ is the index of the topology. The graph topology and the associated Laplacian matrix at $\eta(t)$ are denoted by $\mathcal{G}_{\eta(t)}$ and $L_{\eta(t)}$, respectively.

Lemma 1 ( [14]): If $\mathcal{G}$ contains a spanning tree, then zero is a simple eigenvalue of $L$ with associated right eigenvector $\mathbf{1}_{N}$, and all the other $N-1$ eigenvalues have nonnegative real parts.

Lemma 2 ( [15]): Consider a nonsingular $M$-matrix $L$. There exists a diagonal matrix $G$ such that $G=$ $\operatorname{diag}\left\{g_{1}, \ldots, g_{N}\right\}>0$ and $G L+L^{T} G>0$

Lemma 3 ( [16]): If $a$ and $b$ are nonnegative real numbers and $p$ and $q$ are positive real numbers such that $\frac{1}{p}+\frac{1}{q}=1$, then $a b \leq \frac{a^{p}}{p}+\frac{b^{q}}{q}$, equality holds if and only if $a^{p}=b^{q}$.

\section{Inverse Optimality of Linear Systems}

Consider a linear quadratic regulator problem

$$
\begin{aligned}
& \dot{x}=A x+B u, \\
& J=\int_{0}^{\infty}\left(x^{T} Q x+u^{T} R u\right) d t,
\end{aligned}
$$

where $x \in \mathbb{R}^{n}$ is the state vector, $u \in \mathbb{R}^{m}$ is the control input. $A \in \mathbb{R}^{n \times n}$ and $B \in \mathbb{R}^{n \times m}$ are constant matrices such that $(A, B)$ is controllable and $B$ is of full column rank $m$.

Lemma 4 ([17]): Choosing $Q>0, R>0$, the state feedback control input is given by $u=K x$, where $K=$ $-R^{-1} B^{T} P$ and $P>0$ is a solution to the following Riccati equation

$$
A^{T} P+P A+Q-P B R^{-1} B^{T} P=0 .
$$

Then, the feedback gain $K$ minimizes performance index $J$ and $-K B$ is simple positive definite.

Remark 1: A matrix $H$ is called simple if it is diagonalizable, i.e. there exists nonsingular matrix $T$ such that $T H T^{-1}=\Lambda$, where $\Lambda$ is a diagonal matrix whose diagonal elements are eigenvalues of $H$. As shown in [17], [18], a matrix $H$ is called simple positive definite if it is diagonalizable and its diagonalized form $\Lambda$ is positive definite.

Lemma 5 ( [19]): For the inverse optimal control problem, the feedback control gain $K=-R^{-1} B^{T} P$ is optimal to (1b) and $P>0$ is a solution to the corresponding Riccati equation (2) with some symmetric matrices $R>0$ and $Q>0$, if the following two conditions are hold:

1) $-K B$ is a simple positive definite matrix and $\operatorname{rank}(K B)=\operatorname{rank}(K)$. i.e. there exists some symmetric matrices $R>0$ and $P>0$ such that $K=-R^{-1} B^{T} P$.

2) The feedback control gain $K$ is stabilizing.

\section{Distributed AdAPtive TIME-VARYING FormATION CONTROL PROTOCOL DESIGN}

Consider a group of $N$ identical agents distributed on a directed communication topology, with the dynamics

$$
\dot{x}_{i}=A x_{i}+B u_{i}, \forall i \in\{1, \ldots, N\}
$$

where $x_{i} \in \mathbb{R}^{n}$ are the states, $u_{i} \in \mathbb{R}^{m}$ are the control inputs. $A \in \mathbb{R}^{n \times n}$ and $B \in \mathbb{R}^{n \times m}$ are constant matrices with $\operatorname{rank}(B)=m$.

The dynamics of the leader node, labeled 0 , is described by

$$
\dot{x}_{0}=A x_{0},
$$

where $x_{0} \in \mathbb{R}^{n}$ is the state of the leader. It can be considered as a command generator, which generates the desired target trajectory. The leader can be observed from a subset of agents in a graph. If node $i$ observes the leader, an edge $(0, i)$ is said to exist with weighting gain $a_{i 0}>0$ as a pinned node.

Assumption 1: All possible time-varying switching communication topologies among each agent are assumed to contain a directed spanning tree with the reference generator as the root node.

Then the Laplacian matrix $L_{\eta(t)}$ related to $\mathcal{G}_{\eta(t)}$ can be partitioned as $L_{\eta(t)}=\left[\begin{array}{cc}0 & 0_{1 \times N} \\ L_{2 \eta(t)} & L_{1 \eta(t)}\end{array}\right]$.

The desired formation is specified by the vector $h=$ $\left[h_{1}^{T}, h_{2}^{T}, \ldots, h_{N}^{T}\right]^{T}$ with $h_{i} \in \mathbb{R}^{n}$ being a preset vector known by the corresponding $i^{\text {th }}$ agent. Multi-agent system is said to achieve time-varying formation tracking if for any given bounded initial states

$$
\lim _{t \rightarrow \infty}\left(x_{i}(t)-h_{i}(t)-x_{0}(t)\right)=0 \forall i \in\{1, \ldots, N\} .
$$


Construct the following fully distributed adaptive timevarying formation control protocol

$$
\begin{aligned}
u_{i} & =\left(c_{i}+\rho_{i}\right) K \xi_{i}+\gamma_{i}, \\
\dot{c}_{i} & =\xi_{i}^{T} \Gamma \xi_{i} \quad \forall i \in\{1, \ldots, N\},
\end{aligned}
$$

where $\xi_{i}=\sum_{j=0}^{N} a_{i j}(t)\left[\left(x_{i}-h_{i}\right)-\left(x_{j}-h_{j}\right)\right], c_{i}(t)$ denotes the time varying coupling weight associated with the $i$ th agent with $c_{i}(0) \geq 0, K \in \mathbb{R}^{m \times n}$ and $\Gamma \in \mathbb{R}^{n \times n}$ are the feedback gain matrices, and $\rho_{i}$ and $\gamma_{i}$ are smooth functions to be determined.

Since the matrix $B$ given in (3) is of full rank, there always exists a nonsingular matrix $\left[\tilde{B}^{T}, \bar{B}^{T}\right]^{T}$ with $\tilde{B} \in \mathbb{R}^{m \times n}$ and $\bar{B} \in \mathbb{R}^{(n-m) \times n}$ such that $\tilde{B} B=I_{m}$ and $\bar{B} B=0$.

Theorem 1: Suppose that Assumption 1 holds. If the following formation feasibility condition is satisfied

$$
\bar{B}\left(A h_{i}-\dot{h}_{i}\right)=0 \forall i \in\{1, \ldots, N\},
$$

the time-varying formation specified by $h_{i} \in \mathbb{R}^{n} \forall i \in$ $\{1, \ldots, N\}$ can be achieved under the distributed adaptive control protocol (6) with $K=-R^{-1} B^{T} P, \Gamma=$ $P B R^{-1} B^{T} P, \gamma_{i}=\tilde{B}\left(\dot{h}_{i}-A h_{i}\right)$ and $\rho_{i}=\xi_{i}^{T} P \xi_{i}$, where $P>0$ is a solution to the following Riccati equation:

$$
A^{T} P+P A+Q-P B R^{-1} B^{T} P=0
$$

with $Q>0$ and $R>0$. Furthermore, each coupling weight $c_{i}$ converges to some finite steady-state value.

Proof: Let the global consensus error $\xi=$ $\left[\xi_{1}^{T}, \ldots, \xi_{N}^{T}\right]^{T}$. Define $z_{i}=x_{i}-h_{i} \forall i \in\{1, \ldots, N\}$ and $z=\left[z_{1}^{T}, \ldots, z_{N}^{T}\right]^{T}$. Therefore, the global error vector can be written in a compact form as

$$
\begin{aligned}
\xi & =\left(L_{1 \eta(t)} \otimes I_{n}\right) z+\left(L_{2 \eta(t)} \otimes I_{n}\right) x_{0} \\
& =\left(L_{1 \eta(t)} \otimes I_{n}\right)\left(z-\mathbf{1} \otimes x_{0}\right) .
\end{aligned}
$$

Substituting (6) into (3), the following dynamics of $\xi$ and $c_{i}$ can be obtained

$$
\begin{aligned}
\dot{\xi}= & {\left[I_{N} \otimes A+L_{1 \eta(t)}(C+\rho) \otimes B K\right] \xi } \\
& +\left(L_{1 \eta(t)} \otimes A\right) h-\left(L_{1 \eta(t)} \otimes I_{n}\right) \dot{h}+\left(L_{1 \eta(t)} \otimes B\right) \gamma, \\
\dot{c}_{i}= & \xi_{i}^{T} \Gamma \xi_{i} \quad \forall i \in\{1, \ldots, N\},
\end{aligned}
$$

where $C=\operatorname{diag}\left\{c_{1}, \ldots, c_{N}\right\}, \rho=\operatorname{diag}\left\{\rho_{1}, \ldots, \rho_{N}\right\}$, and $\gamma=\left[\gamma_{1}^{T}, \gamma_{2}^{T}, \ldots, \gamma_{N}^{T}\right]^{T}$.

Consider the following Lyapunov function candidate

$$
V_{1}=\sum_{i=1}^{N} \frac{1}{2} g_{i}\left(2 c_{i}+\rho_{i}\right) \rho_{i}+\frac{1}{2} \sum_{i=1}^{N} g_{i}\left(c_{i}-\alpha\right)^{2}
$$

where $G=\operatorname{diag}\left\{g_{1}, \ldots, g_{N}\right\}$ is a positive definite matrix such that $G L_{1 \eta(t)}+L_{1 \eta(t)}^{T} G>0$, and $\alpha$ is a positive constant to be determined later. According to Lemma 1 and the fact that $L_{1 \eta(t)}$ is a nonsingular $M$-matrix, the existence of such a positive definite matrix $G$ can be guaranteed from Lemma 2 . Because $c_{i}(0)>0$, it follows from $\dot{c}_{i}(t) \geq 0$ that $c_{i}(t)>0$ for any $t>0$. Then, it is easy to conclude that $V_{1}$ is positive definite.
Thus, the time derivative of $V_{1}$ along the trajectory of (10) is obtained as

$$
\begin{aligned}
\dot{V}_{1} & =\sum_{i=1}^{N}\left[g_{i}\left(c_{i}+\rho_{i}\right) \dot{\rho}_{i}+g_{i} \rho_{i} \dot{c}_{i}\right]+\sum_{i=1}^{N} g_{i}\left(c_{i}-\alpha\right) \dot{c}_{i} \\
& =\sum_{i=1}^{N} 2 g_{i}\left(c_{i}+\rho_{i}\right) \xi_{i}^{T} P \dot{\xi}_{i}+\sum_{i=1}^{N} g_{i}\left(\rho_{i}+c_{i}-\alpha\right) \dot{c}_{i}
\end{aligned}
$$

Note that

$$
\sum_{i=1}^{N} g_{i}\left(\rho_{i}+c_{i}-\alpha\right) \dot{c}_{i}=\xi^{T}[(C+\rho-\alpha I) G \otimes \Gamma] \xi,
$$

and

$$
\begin{array}{rl}
\sum_{i=1}^{N} & 2 g_{i}\left(c_{i}+\rho_{i}\right) \xi_{i}^{T} P \dot{\xi}_{i}=2 \xi^{T}[(C+\rho) G \otimes P] \dot{\xi} \\
= & \xi^{T}\left[(C+\rho) G \otimes\left(P A+A^{T} P\right)\right. \\
& \left.-(C+\rho)\left(G L_{1 \eta(t)}+L_{1 \eta(t)}^{T}\right) G(C+\rho) \otimes \Gamma\right] \xi \\
& +2 \xi^{T}\left[(C+\rho) G L_{1 \eta(t)} \otimes P A\right] h \\
& -2 \xi^{T}\left[(C+\rho) G L_{1 \eta(t)} \otimes P\right] \dot{h} \\
& +2 \xi^{T}\left[(C+\rho) G L_{1 \eta(t)} \otimes P B\right] \gamma \\
\leq & \xi^{T}\left[(C+\rho) G \otimes\left(P A+A^{T} P\right)\right. \\
& \left.-\lambda_{0}^{m i n}(C+\rho)^{2} \otimes \Gamma\right] \xi \\
& +2 \xi^{T}\left[(C+\rho) G L_{1 \eta(t)} \otimes P A\right] h \\
& -2 \xi^{T}\left[(C+\rho) G L_{1 \eta(t)} \otimes P\right] \dot{h} \\
& +2 \xi^{T}\left[(C+\rho) G L_{1 \eta(t)} \otimes P B\right] \gamma
\end{array}
$$

where $\lambda_{0}^{\min }$ represents the minimum of the smallest positive eigenvalues of $G L_{1 \eta(t)}+L_{1 \eta(t)}^{T} G$ for all $\mathcal{G}_{\eta(t)}$.

If condition (7) holds, then for all $i \in\{1, \ldots, N\}$

$$
\bar{B} A h_{i}-\bar{B} \dot{h}_{i}+\bar{B} B \gamma_{i}=0 .
$$

By letting $\gamma_{i}=\tilde{B} \dot{h}_{i}-\tilde{B} A_{r} h_{i}$, it follows that

$$
\tilde{B} A h_{i}-\tilde{B} \dot{h}_{i}+\tilde{B} B \gamma_{i}=0 \text {. }
$$

From (15) and (16) and the fact that $\left[\tilde{B}^{T}, \bar{B}^{T}\right]^{T}$ is nonsingular, one gets

$$
A h_{i}-\dot{h}_{i}+B \gamma_{i}=0
$$

which means that

$$
\left(I_{N} \otimes A\right) h-\left(I_{N} \otimes I_{N}\right) \dot{h}+\left(I_{N} \otimes B\right) \gamma=0 .
$$

Pre-multiplying the both sides of (18) by $(C+\rho) G L_{1 \eta(t)} \otimes P$ yields

$$
\begin{aligned}
{\left[(C+\rho) G L_{1 \eta(t)} \otimes\right.} & P A] h-\left[(C+\rho) G L_{1 \eta(t)} \otimes P\right] \dot{h} \\
& +\left[(C+\rho) G L_{1 \eta(t)} \otimes P B\right] \gamma=0 .
\end{aligned}
$$

Therefore, we obtain

$$
\begin{gathered}
\dot{V}_{1} \leq \xi^{T}\left[(C+\rho) G \otimes\left(P A+A^{T} P+\Gamma\right)\right. \\
\left.-\left(\lambda_{0}^{\min }(C+\rho)^{2}+\alpha G\right) \otimes \Gamma\right] \xi .
\end{gathered}
$$




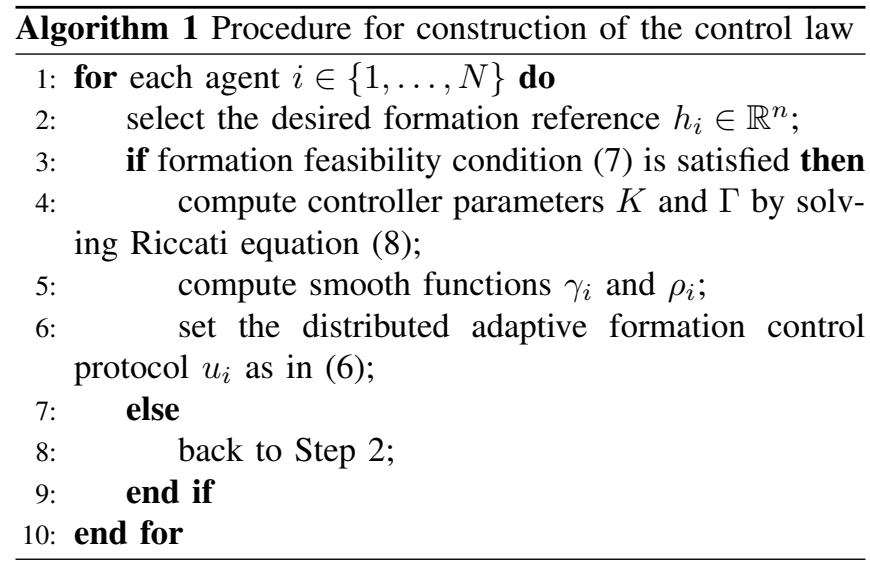

From Lemma 3, we have

$$
\begin{aligned}
& \left.-\xi^{T}\left(\lambda_{0}^{\min }(C+\rho)^{2}+\alpha G\right) \otimes \Gamma\right] \xi \\
\leq & -2 \xi^{T}\left[\sqrt{\lambda_{0}^{\min } \alpha G}(C+\rho) \otimes \Gamma\right] \xi .
\end{aligned}
$$

Selecting $\alpha \geq \frac{\max _{i \in\{1, \ldots, N\}} g_{i}}{\lambda_{0}^{\text {min }}}$ and substituting (21) into (20) yields

$$
\dot{V}_{1} \leq \xi^{T}\left[(C+\rho) G \otimes\left(P A+A^{T} P-\Gamma\right)\right] \xi .
$$

Define $\zeta=(\sqrt{(C+\rho) G} \otimes I) \xi$. Therefore, it follows from (22) that

$$
\begin{aligned}
\dot{V}_{1} & \leq \zeta^{T}\left[I_{N} \otimes\left(P A+A^{T} P-P B R^{-1} B^{T} P\right)\right] \zeta \\
& \leq 0
\end{aligned}
$$

where the last inequality comes immediately from the Riccati equation (8). Since $V_{1}(t) \geq 0$ and $\dot{V}_{1}(t) \leq 0, V_{1}(t)$ is bounded, and so is each $c_{i}$. Noting that $\dot{c}_{i} \geq 0$, it can be concluded that each coupling weight $c_{i}$ converges to some finite value. $\dot{V}_{1} \equiv 0$ implies $\zeta \equiv 0$, which in turn implies that $\xi \equiv 0$. By using LaSalle's invariance principle [20], we have the formation tracking error $\xi$ asymptotically converges to zero. Therefore, the fully distributed time-varying formation tracking problem is solved.

Remark 2: It should be noted that the formation control problem reduces to a consensus problem when $h_{i}=0 \forall i \in$ $\{1, \ldots, N\}$, such that the protocol shown in [21] can be viewed as a special case of the result in the current paper.

Remark 3: The proposed distributed adaptive formation tracking protocol in this paper is different from that in [12], where it is necessary to calculate the minimum positive eigenvalue of the Laplacian matrix of the communication topology. Our distributed cooperative controller allows each agent only to access the information from its neighbors such that the proposed controller is fully distributed regardless of global information.

With the above analysis, the procedure to construct the control law $u_{i}$ is given in Algorithm 1 .

\section{Analysis of LQR-Based Global Optimality}

In this section, the essential constraints on graph topology for global optimality of the proposed distributed control (6) is provided.
Theorem 2: Under the Assumption 1 and condition (7), the proposed distributed adaptive formation control protocol minimizes the certain LQR performance index

$$
J=\int_{0}^{\infty}\left(\xi^{T} \bar{Q} \xi+v^{T} \bar{R} v\right) d t
$$

with $\bar{Q}=\bar{Q}^{T}>0, \bar{R}=\bar{R}^{T}>0$, and $v=\left(I_{N} \otimes\right.$ $B)\left(L_{1 \eta(t)}(C+\rho) \otimes K\right) \xi$, if the Laplacian matrix of the graph is simple.

Proof: If the graph Laplacian is simple, it is easy to verify that $L_{1 \eta(t)}$ is a simple positive definite matrix. Since $(C+\rho)$ becomes a constant positive definite matrix after the time-varying formation is achieved, it can be concluded that $L_{1 \eta(t)}(C+\rho)$ is simple positive definite. Then the dynamics of the global error system can be given by

$$
\begin{aligned}
\dot{\xi} & =\left[I_{N} \otimes A+\left(I_{N} \otimes B\right)\left(L_{1 \eta(t)}(C+\rho) \otimes K\right)\right] \xi \\
& =\left(I_{N} \otimes A\right) \xi+\left(I_{N} \otimes B\right) v,
\end{aligned}
$$

Note that $-K B$ is simple positive definite from Lemma 4 , we have $\operatorname{rank}(K B)=\operatorname{rank}(K)$. It follows that $L_{1 \eta(t)}(C+$ $\rho) \otimes-K B$ is simple positive definite, such that

$$
\begin{aligned}
& \operatorname{rank}\left[\left(L_{1 \eta(t)}(C+\rho) \otimes K\right)\left(I_{N} \otimes B\right)\right] \\
= & \operatorname{rank}\left[L_{1 \eta(t)}(C+\rho)\right] \operatorname{rank}(K B) \\
= & \operatorname{rank}\left[L_{1 \eta(t)}(C+\rho) \otimes K\right]
\end{aligned}
$$

It can be seen that Lemma 5 is satisfied. Therefore, error dynamics (25) is optimal for the following global performance index

$$
J=\int_{0}^{\infty}\left(\xi^{T} \bar{Q} \xi+v^{T} \bar{R} v\right) d t
$$

with $\bar{Q}=\bar{Q}^{T}>0$ and $\bar{R}=\bar{R}^{T}>0$.

According to [22], one obtains the optimal performance index $\bar{J}=\min \int_{0}^{\infty}\left(\xi^{T} \bar{Q} \xi+v^{T} \bar{R} v\right) d t=\xi(0)^{T} \bar{P} \xi(0)$, and

$$
\begin{array}{r}
\bar{Q}-\bar{P}\left(I_{N} \otimes B\right) \bar{R}^{-1}\left(I_{N} \otimes B\right)^{T} \bar{P}+ \\
\bar{P}\left(I_{N} \otimes A\right)+\left(I_{N} \otimes A\right)^{T} \bar{P}=0 .
\end{array}
$$

Furthermore, $\bar{K}=L_{1 \eta(t)}(C+\rho) \otimes K$ should have the same form as $K$. Define $L_{\sigma(t)}=L_{1 \eta(t)}(C+\rho)$, one has

$$
\begin{aligned}
\bar{K} & =L_{1 \eta(t)}(C+\rho) \otimes K \\
& =-L_{\sigma(t)} \otimes R^{-1} B^{T} P \\
& =-\left(I_{N} \otimes R\right)^{-1}\left(I_{N} \otimes B\right)^{T}\left(L_{\sigma(t)} \otimes P\right) .
\end{aligned}
$$

By letting $\bar{R}=I_{N} \otimes R$ and $\bar{P}=L_{\sigma(t)} \otimes P, \bar{Q}$ can be obtained by (28) as

$$
\bar{Q}=\left(L_{\sigma(t)}^{2}-L_{\sigma(t)}\right) \otimes \Gamma+L_{\sigma(t)} \otimes Q .
$$

Remark 4: Different from the results shown in [19], we prove that the global optimality of the distributed adaptive control can be achieved in directed graphs with simple graph Laplacian matrices. One way to find such a new class of digraphs is to ensure that there exists no cycles in the topologies, please refer to [17], [18] for more details. 


\section{Simulation Results}

In this section, we present some simulation results to validate the performance of the proposed distributed adaptive time-varying formation control protocol.

Consider a multi-agent system with eight agents and the dynamics of each agent are described by (3) with

$$
A=\left[\begin{array}{ccc}
0 & 1 & 1 \\
1 & 2 & 1 \\
-2 & -6 & -3
\end{array}\right], B=\left[\begin{array}{l}
0 \\
0 \\
1
\end{array}\right]
$$

The switching directed interaction topologies among the eight agents are shown in Fig. 1, where the leader agent 0 provides the formation reference signal and the directed topology is switched every 2.5 seconds in sequence. It can be seen that all these graphs have simple Laplacian matrices. Let $\tilde{B}=\left[\begin{array}{lll}0 & 0 & 1\end{array}\right]$ and $\bar{B}=\left[\begin{array}{lll}1 & 0 & 0 \\ 0 & 1 & 0\end{array}\right]$ such that $\tilde{B} B=$ $I$ and $\bar{B} B=0$. Recall that $h_{i} \in \mathbb{R}^{n}$ is the formation offset vector with respect to the formation reference $x_{0} \in \mathbb{R}^{n}$, which is given by

$$
h_{i}=\left[\begin{array}{c}
15 \sin \left(t+\frac{2(i-1) \pi}{8(8-1)}\right) \\
-15 \cos \left(t+\frac{2(i-1) \pi}{8}\right) \\
30 \cos \left(t+\frac{2(i-1) \pi}{8}\right)
\end{array}\right],(\text { for } i \in\{1,2, \ldots, 8\}) .
$$

It can be verified that the formation tracking feasibility condition (7) in Theorem 1 is satisfied. Thus, if the predefined time-varying formation $h_{i}(t)$ is achieved, the eight agents will form a parallel octagon and keep rotating around the leader with an angular velocity of $1 \mathrm{rad} / \mathrm{s}$. Then according to Algorithm 1, the controller gains can be calculated as

$$
\begin{gathered}
K=\left[\begin{array}{ccc}
-43.9086 & -131.5675 & -33.8775
\end{array}\right], \\
\Gamma=\left[\begin{array}{ccc}
1.9276 & 5.7758 & 1.4872 \\
5.7784 & 17.3143 & 4.4583 \\
1.4885 & 4.4601 & 1.1484
\end{array}\right]
\end{gathered}
$$

by setting $Q=\left[\begin{array}{lll}1 & 0 & 0 \\ 0 & 1 & 0 \\ 0 & 0 & 1\end{array}\right]$ and $R=0.001$. Based on the solution of corresponding algebraic Riccati equation, $\rho_{i}$ is given by

$$
\rho_{i}=\xi_{i}^{T}\left[\begin{array}{lll}
1.0036 & 0.5518 & 0.0439 \\
0.5518 & 4.1963 & 0.1316 \\
0.0439 & 0.1316 & 0.0339
\end{array}\right] \xi_{i} .
$$

Let initial value of coupling weight $c_{i}(0)=0$ for all $i \in$ $\{1, \ldots, 8\}$ and initial states of each agent be pseudorandom values with a uniform distribution on the interval $(-0.5,0.5)$.

The state trajectory snapshots of all agents in the multiagent system is illustrated in Fig. 2. The state response of each agent is shown in Fig. 3. The values of the coupling weight $c_{i}$ and time-varying formation error $\xi_{i}$ are depicted in Fig. 4 and Fig. 5 respectively. From all these figures, it can be seen that all eight agents form a parallel octagon while keeping rotating around the virtual leader which lies in the center of the parallel octagon after 5 seconds. The coupling weights are bounded and converge to some finite positive constants. Therefore, it is concluded that the desired timevarying formation tracking is achieved under the proposed fully distributed adaptive protocol (6).

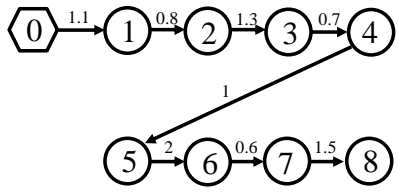

(a)

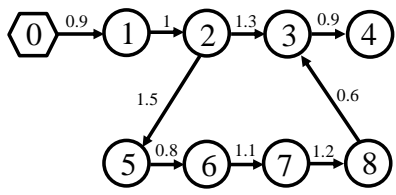

(c)

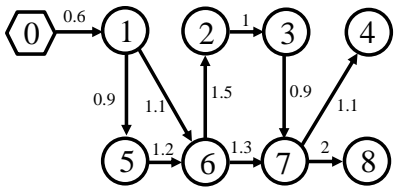

(b)
Fig. 1. Switching directed interaction topologies.

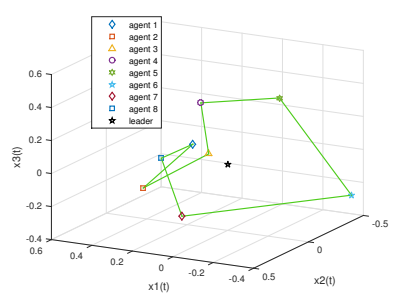

(a)

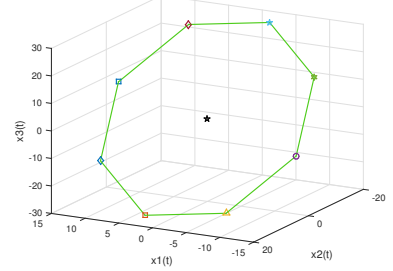

(c)

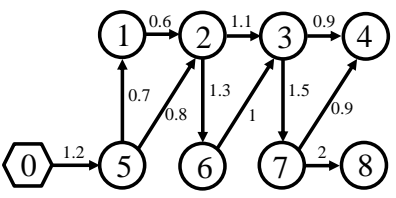

(d)

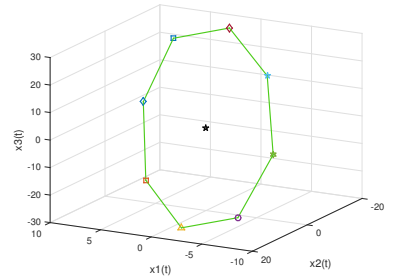

(b)

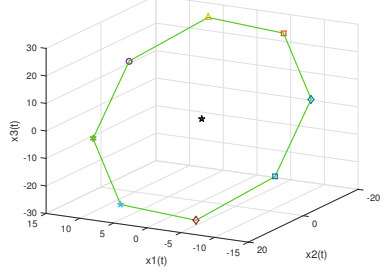

(d)
Fig. 2. State snapshots of all the agents: (a) $t=0$ s. (b) $t=3$ s. (c) $t=10$ s. (d) $t=15 \mathrm{~s}$
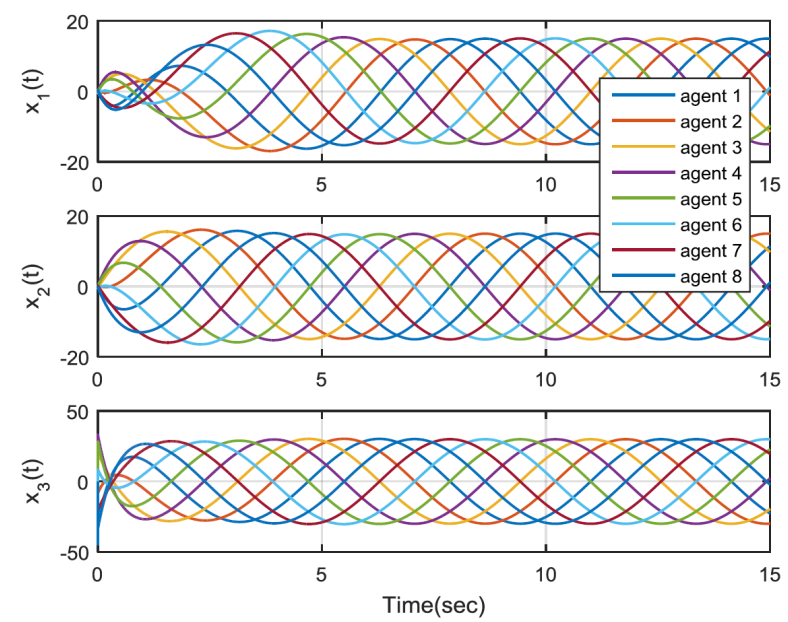

Fig. 3. State responses of the multi-agent systems

\section{CONCLUSIONS}

In this paper, a fully distributed time-varying formation tracking problem of general linear multi-agent systems over 


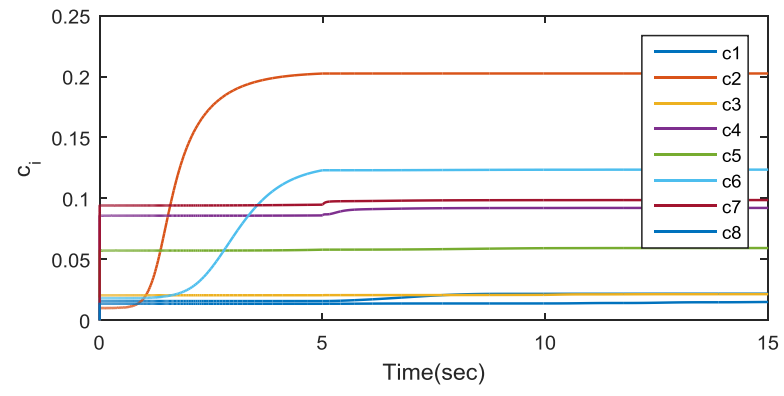

Fig. 4. The coupling weight $c_{i}$.
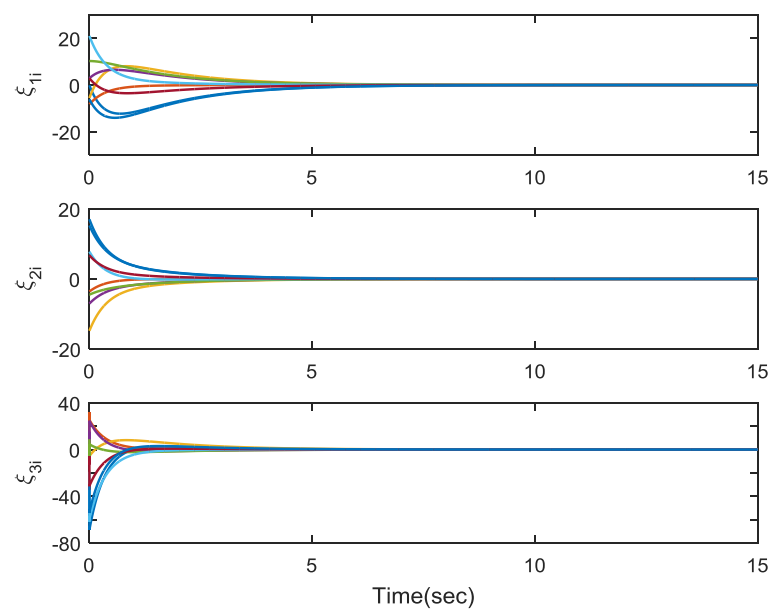

Fig. 5. The time-varying formation tracking error.

switching directed graphs has been solved. An adaptive timevarying formation tracking protocol was first proposed based on relative state information, which made the proposed control design fully distributed regardless of global information. Then, an algorithm was presented to construct the control law by testing formation feasibility condition and solving algebraic Riccati equation. The stability of the proposed algorithm was proved by Lyapunov theory. Moreover, it was shown that the proposed formation protocol can achieve LQR-based global optimality via inverse optimal control theory. Finally, the effectiveness of the proposed strategy was verified by simulation results.

Future works will take time delays and heterogeneous dynamics of multi-agent systems [23] into consideration, and robust methods such as [24], [25] will be exploited in the design of the distributed control protocol.

\section{REFERENCES}

[1] J. Wang, A. Lanzon, and I. R. Petersen, "Robust output feedback consensus for networked negative-imaginary systems," IEEE Transactions on Automatic Control, vol. 60, no. 9, pp. 2547-2552, 2015.

[2] C. Yang, F. Chen, L. Xiang, and W. Lan, "Distributed rendezvous and tracking for multiple unicycles with heterogeneous input disturbances," International Journal of Robust and Nonlinear Control, vol. 27, no. 9, pp. 1589-1606, 2017.

[3] Z. Li, Z. Duan, W. Ren, and G. Feng, "Containment control of linear multi-agent systems with multiple leaders of bounded inputs using distributed continuous controllers," International Journal of Robust and Nonlinear Control, vol. 25, no. 13, pp. 2101-2121, 2015.
[4] J. Hu and A. Lanzon, "An innovative tri-rotor drone and associated distributed aerial drone swarm control," Robotics and Autonomous Systems, vol. 103, pp. 162-174, 2018.

[5] W. Ren, "Consensus strategies for cooperative control of vehicle formations," IET Control Theory \& Applications, vol. 1, no. 2, pp. 505-512, 2007.

[6] Z. Wu, Z. Guan, X. Wu, and T. Li, "Consensus based formation control and trajectory tracing of multi-agent robot systems," Journal of Intelligent \& Robotic Systems, vol. 48, no. 3, pp. 397-410, 2007.

[7] H. Du, S. Li, and X. Lin, "Finite-time formation control of multiagent systems via dynamic output feedback," International Journal of Robust and Nonlinear Control, vol. 23, no. 14, pp. 1609-1628, 2013.

[8] J. A. Fax and R. M. Murray, "Information flow and cooperative control of vehicle formations," IEEE Transactions on Automatic Control, vol. 49, no. 9, pp. 1465-1476, 2004.

[9] M. Porfiri, D. G. Roberson, and D. J. Stilwell, "Tracking and formation control of multiple autonomous agents: A two-level consensus approach," Automatica, vol. 43, no. 8, pp. 1318-1328, 2007.

[10] M. Turpin, N. Michael, and V. Kumar, "Decentralized formation control with variable shapes for aerial robots," in Proceeding of the IEEE International Conference on Robotics and Automation (ICRA), 2012, pp. 23-30.

[11] X. Dong, J. Xiang, L. Han, Q. Li, and Z. Ren, "Distributed timevarying formation tracking analysis and design for second-order multiagent systems," Journal of Intelligent \& Robotic Systems, vol. 86, no. 2, pp. 277-289, 2017.

[12] X. Dong and G. Hu, "Time-varying formation control for general linear multi-agent systems with switching directed topologies," $\mathrm{Au}$ tomatica, vol. 73, pp. 47-55, 2016.

[13] R. Wang, X. Dong, Q. Li, and Z. Ren, "Distributed adaptive timevarying formation for multi-agent systems with general high-order linear time-invariant dynamics," Journal of the Franklin Institute, vol. 353, no. 10, pp. 2290-2304, 2016.

[14] W. Ren and R. W. Beard, "Consensus seeking in multiagent systems under dynamically changing interaction topologies," IEEE Transactions on Automatic Control, vol. 50, no. 5, pp. 655-661, 2005.

[15] H. Zhang, F. L. Lewis, and Z. Qu, "Lyapunov, adaptive, and optimal design techniques for cooperative systems on directed communication graphs," IEEE Transactions on Industrial Electronics, vol. 59, no. 7, pp. 3026-3041, 2012.

[16] D. S. Bernstein, Matrix mathematics: Theory, facts, and formulas with application to linear systems theory. Princeton University Press Princeton, 2005, vol. 41.

[17] H. Zhang, T. Feng, G.-H. Yang, and H. Liang, "Distributed cooperative optimal control for multiagent systems on directed graphs: An inverse optimal approach," IEEE Transactions on Cybernetics, vol. 45, no. 7, pp. 1315-1326, 2015.

[18] K. H. Movric and F. L. Lewis, "Cooperative optimal control for multiagent systems on directed graph topologies," IEEE Transactions on Automatic Control, vol. 59, no. 3, pp. 769-774, 2014.

[19] Z. Li and Z. Ding, "Fully distributed adaptive consensus control of multi-agent systems with lqr performance index," in Proceedings of IEEE Conference on Decision and Control (CDC), 2015, pp. 386-391.

[20] H. K. Khalil, "Noninear systems," Prentice-Hall, New Jersey, vol. 2, no. 5, pp. 5-1, 1996.

[21] Y. Lv, Z. Li, Z. Duan, and G. Feng, "Novel distributed robust adaptive consensus protocols for linear multi-agent systems with directed graphs and external disturbances," International Journal of Control, vol. 90, no. 2, pp. 137-147, 2017.

[22] B. D. Anderson and J. B. Moore, Optimal control: linear quadratic methods. Courier Corporation, 2007.

[23] J. Wang, A. Lanzon, and I. R. Petersen, "Robust cooperative control of multiple heterogeneous negative-imaginary systems," Automatica, vol. 61, pp. 64-72, 2015.

[24] A. Lanzon, A. Freddi, and S. Longhi, "Flight control of a quadrotor vehicle subsequent to a rotor failure," AIAA Journal of Guidance, Control and Dynamics, vol. 37, no. 2, pp. 580-591, 2014.

[25] A. Lanzon and G. Papageorgiou, "Distance measures for uncertain linear systems: A general theory," IEEE Transactions on Automatic Control, vol. 54, no. 7, pp. 1532-1547, 2009. 